\title{
Implementation of Linear Prediction Models for Lossless Compression of Hyperspectral Images in Novel Parallel Environments
}

\author{
Jarno Mielikäinen and Pekka Toivanen \\ Lappeenranta University of Technology, \\ P.O. Box 20, \\ 53851 Lappeenranta, \\ Finland \\ mielikai@lut.fi, ptoivane@lut.fi
}

\begin{abstract}
This paper presents the implementation of a new method for lossless compression of hyperspectral images for novel parallel environments. The method in question is an interband version of the linear prediction approach for hyperspectral images. The interband linear prediction method consists of two stages: predictive decorrelation that produces residuals and the entropy coding of the residuals. The results and comparisons with other methods are discussed. The speedup of the thread version is almost linear with respect to the number of processors.
\end{abstract}

\section{Introduction}

Technological progress has made a huge amounts of data available at increasing spatial and spectral resolutions; therefore compression of hyperspectral data is a subject of active research. In some fields, the original quality of a hyperspectral image cannot be compromised and in these cases lossless compression is mandatory.

A novel compression scheme for the lossless compression of hyperspectral images was introduced in [?]. This paper presents an implementation of that method for novel parallel environments. PCs that use shared memory and enable thread level parallelism are called Symmetric Multi-Processor (SMP) PCs. By connecting these SMP PCs into a cluster that uses message passing for communication, it is possible to create an economical distributed parallel environment using commodity components. We consider these clusters to be novel parallel environments.

\section{Linear Prediction for the Lossless Compression of Hyperspectral Images}

The basic idea, see Figure 1, is to use a linear combination of a small number of pixels in the causal neighborhood of the current pixel as the predictor. From 
here on, the causal neighborhood of the current pixel will be referred to as the causal set. The coefficients for linear prediction are the optimal coefficients that use the Least Squares (LS) estimation approach for pixels that are in the larger causal neighborhood of the current pixel, known as the sample set. The residuals between the predicted and real values are then calculated. The pixels, which do not have a sufficiently large causal environment and, therefore, cannot be predicted, are simply coded as difference between the values in the same spatial position between consecutive bands.

The residuals after the linear prediction of each band are entropy-coded separately using a range coder [?].

An estimate $p_{x, y, z}^{\prime}$ for the current pixel $p_{x, y, z}$ at location $(x, y, z)$ is calculated using linear prediction as

$$
p_{x, y, z}^{\prime}=\sum_{k=0}^{O} \sum_{j=0}^{T} \sum_{i=L_{j, k}}^{R_{j, k}} a_{i, j, k} p_{x-i, y-j, z+k},
$$

where $a_{i, j, k}$ is a prediction coefficient of the pixel at location $(i, j, k), O$ the number of bands and $T$ the number of rows that take part in the causal set. $L(j, k)$ and $R(j, k)$ are the left and right delimiters as a functions of the rows and bands that finally specify the causal set. An example of a causal set that uses three bands can be seen in Figure 2.

We will relabel the terms $p_{x, y, z}, p_{x-i, y-j, z+k}$ and $a_{i, j, k}$ in Equation 1 as $P_{0}$, $\ldots, P_{K}$ and $A_{1}, \ldots, A_{K}$, where $K$ is the size of the causal set. Using this notation, an estimate $P_{0}^{\prime}$ for the current pixel $P_{0}$ from Equation 1 can be rewritten as

$$
P_{0}^{\prime}=\left[\begin{array}{lll}
A_{1} & \ldots & A_{K}
\end{array}\right] \cdot\left[\begin{array}{lll}
P_{1} & \ldots & P_{K}
\end{array}\right]^{T} .
$$

At the same time, there are $M=W_{L}+\left(W_{L}+W_{R}+1\right) \cdot S$ observations in the sample set. Parameters $W_{l}, W_{r}, S$ are on the left and right and at the top of the sample set in Figure 3. Thereby, we have $M$ equations and $K$ unknown variables.

By arranging the pixel values in the matrix form so that each row, $j$, contains the pixel values used in the prediction of the $j$ th pixel in the sample set, the index $j$ in matrix element $P_{i, j}$ refers to the $j$ th value in the sample set,

$$
P=\left[\begin{array}{cccc}
P_{1,1} & P_{2,1} & \ldots & P_{K, 1} \\
P_{1,2} & P_{2,2} & \ldots & P_{K, 2} \\
\ldots & \ldots & \ldots & \ldots \\
P_{1, M-1} & P_{2, M-1} & \ldots & P_{K, M-1} \\
P_{1, M} & P_{2, M} & \ldots & P_{K, M}
\end{array}\right] .
$$

Let vector $Y$ contain the real pixel values in the sample set

$$
Y=\left[\begin{array}{c}
P_{0,1} \\
P_{0,2} \\
\cdots \\
P_{0, M-1} \\
P_{0, M}
\end{array}\right]
$$


The linear prediction, $Y^{\prime}$, for the real values, $Y$, in the sample set is

$$
Y^{\prime}=P \cdot A
$$

With this information, we can calculate the optimal coefficients $A=\left(A_{1}, \ldots, A_{K}\right)^{T}$ so that $\left(Y^{\prime}-Y\right)^{T}\left(Y^{\prime}-Y\right)$ is minimized. [?]

The coefficients for the least squares estimate are

$$
A=\left(P^{T} P\right)^{-1} P^{T} Y .
$$

When the causal set contains only one value $(K=1)$, Equation 6 simplifies to

$$
A=\frac{I \cdot Y}{I \cdot P}
$$

where $I=\left(\begin{array}{lllll}1 & 1 & \ldots & 1 & 1\end{array}\right)$.

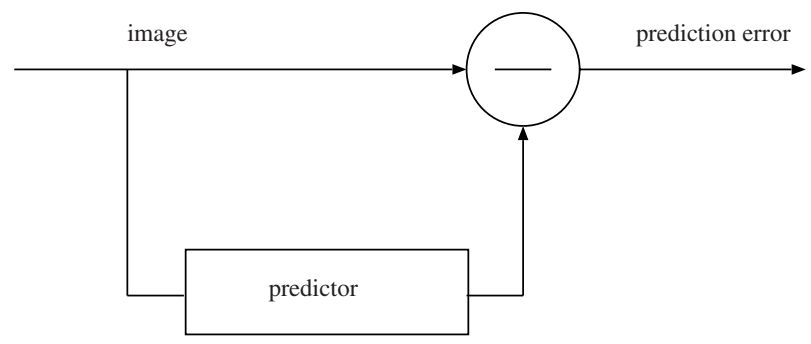

Fig. 1. Prediction of the current pixel.

\section{Parallel implementation}

The thread implementation of compression is quite straight forward: the workload is divided so that each processor gets as equal number of the bands as possible and then the processor performs the prediction and entropy coding on each band that is assigned to it.

In the distributed implementation of compression, each slave PC first sends information to the master about the number of processors in that $\mathrm{PC}$ and the bogomips number that gives some kind of bogus number about the performance of the processors. The workload is distributed proportionally to the cumulative bogomips sum on each PC. The master then sends the bands to the slaves and the slaves return the entropy coded bands when they are done. Inside the PC, the thread level distribution is performed according to the principles laid down in the previous paragraph on the thread implementation of compression. 


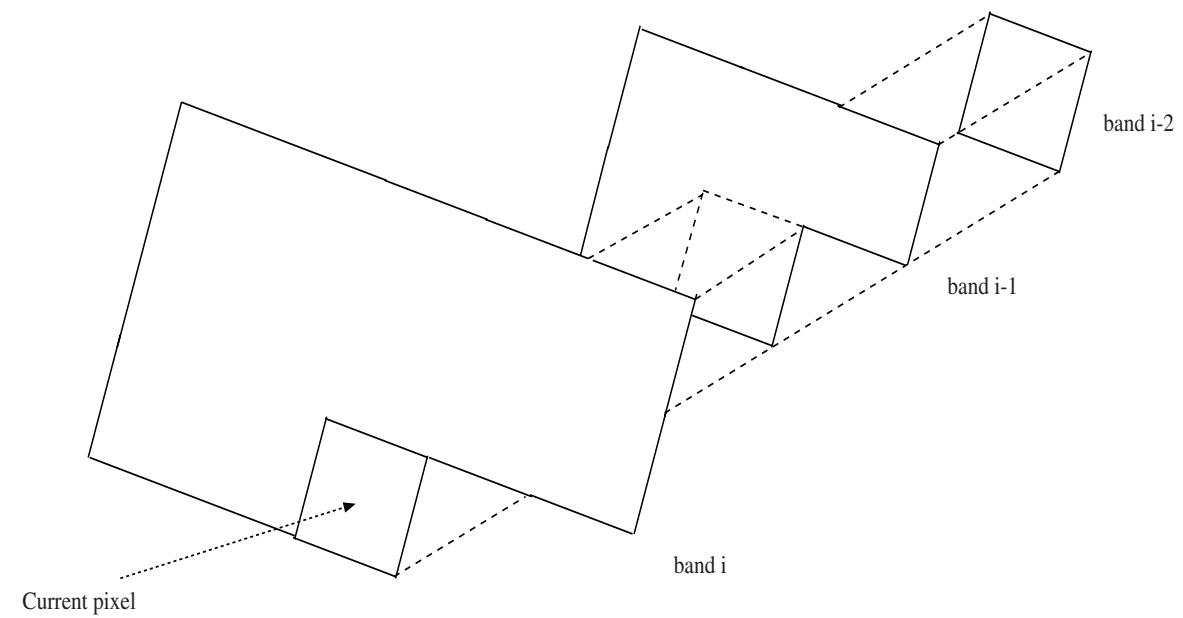

Fig. 2. An example of a causal set.

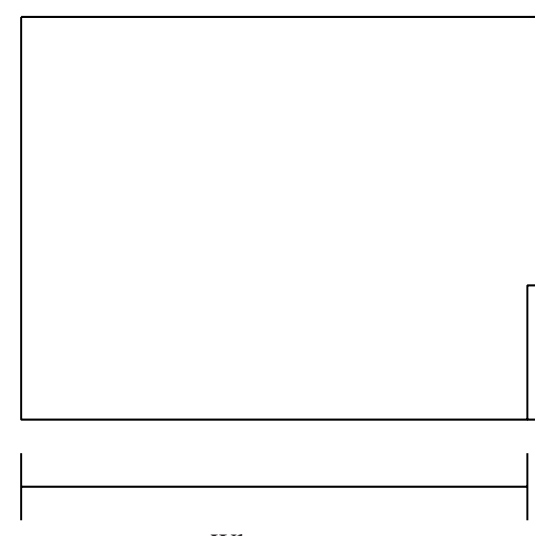

W1
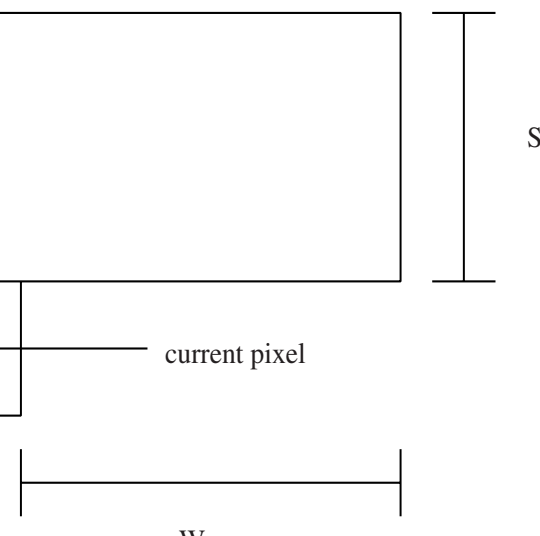

Fig. 3. A sample set with its left $(W l)$, right $(W r)$, and top $(S)$ parameters. 
The thread implementation of decompression is performed by first distributing the workload for decompression, just like in the compression part. After the decoding is done the prediction is done in the form of a pipeline process. When row $r$ in band $b$ has been predicted, and therefore the original row $r$ in band $b$ has been recovered, row $r$ in band $b+1$ can be predicted, and so on.

\section{Compression Results}

In [?], it was shown that when only one value from the previous band was used in the spectral dimension, the compression ratio turned out to be higher than when the causal set also contained values from the spatial direction. This was also the fastest method, since it had fewer prediction coefficients than did the other methods. Therefore, we used only the pixel in the same spatial position from the previous band in the Equation 1. Thus, the prediction Equation 1 can in fact be rewritten as

$$
p_{x, y, z}^{\prime}=a p_{x, y, z-1}
$$

where $a$ is a prediction coefficient.

The performance of the method was measured in the form of an average using 13 images from the Airborne Visible InfraRed Imaging Spectrometer (AVIRIS) '97 image set [?]. The spatial size of the images was 614 by 512, and they contained 224 bands. AVIRIS covers the solar reflected portion of the electromagnetic spectrum $0.41 \mu \mathrm{m}$ to $2.45 \mu \mathrm{m}$ in $10 \mathrm{~nm}$ bands. The AVIRIS instrument consists of four spectrometers that view a 20-square-meter spot on the ground from a flight altitude of $20 \mathrm{~km}$. This spot is simultaneously viewed in 224 spectral bands. By moving the spectrometers perpendicular to the aircrafts direction, a spatial image of 614 pixels is formed. [?][?]

In Table 1, the compression ratios are compared to various other methods. The compression ratio is measured as the size of the original file divided by the size of the compressed file. The following abbreviations are used in Table 1:

- DCT: spatial/spectral DCT [?]

- VQ-diff: spectral difference of Vector Quantizer residual [?]

- LP: linear prediction (our method)

Table 1. The average compression ratios for 13 AVIRIS '97 images.

\begin{tabular}{|c|c|c|}
\hline DCT & VQ-diff & LP \\
\hline 2.72 & 3.06 & 3.12 \\
\hline
\end{tabular}

It can be seen that our method does, in fact, give higher compression ratios than do the other two methods. 


\section{Performance Evaluation}

The speedup of a compression method on an SMP PC can be seen in Table ??. The speedup is measured as

$$
\frac{\text { time }(1 \text { processor })}{\text { time }(\text { p processors })}
$$

where $p$ is the number of processors. When the speedup is calculated in an heterogeneous environment, we define it as follows

$$
\frac{(\text { time }(\text { processor } 1)+\text { time }(\text { processor } 2)+\ldots+\text { time }(\text { processor } p)) / p}{\text { time }(\text { p processors })} \text {. }
$$

The PC that was used in the test is a quad processor $700 \mathrm{MHz}$ Pentium Xeon. From the results in the Table ??, it can be seen that the speedup is almost linear.

Table 2. The speedup of a compression method on an SMP PC.

\begin{tabular}{|c|c|}
\hline \# of processors & speedup \\
\hline 2 & 1.78 \\
3 & 2.41 \\
4 & 3.18 \\
\hline
\end{tabular}

The speedup of a compression method in a distributed environment can be seen in Table ??. The first four PC are 1-GHz dual-processor Pentium III PCs and the last four $800 \mathrm{MHz}$ dual-processor Pentium III PCs. All the PCs were connected using a gigabit Ethernet.

Table 3. The speedup of a compression method in a distributed environment

\begin{tabular}{|c|c|}
\hline \# of dual processor PCs & speedup \\
\hline 1 & 1.81 \\
2 & 2.74 \\
3 & 3.25 \\
4 & 3.66 \\
5 & 4.00 \\
6 & 4.24 \\
7 & 4.32 \\
8 & 4.27 \\
\hline
\end{tabular}

The speedup of a decompression method on an SMP PC can be seen in Table ??. The same $700-\mathrm{MHz}$ Pentium Xeon was used as it was in compression. 
It is quite interesting to note that the speedup in this case is actually better than it was in the case of compression, which just divided the bands among the processors.

Table 4. The speedup of a decompression method on an SMP PC.

\begin{tabular}{|c|c|}
\hline \# of processors & speedup \\
\hline 2 & 1.89 \\
3 & 2.72 \\
4 & 3.44 \\
\hline
\end{tabular}

\section{Conclusions}

The results were very promising: the compression ratio was higher than with competing methods. Thus, the results confirm our suspicion that the image is not stationary but its statistics vary along the image. Therefore, our method that recalculates the prediction coefficient for each pixel is able to adapt to the local statistical variations in the image.

Although the scaling of the distributed version was rather limited due to the communication overhead, the scaling of the thread version is almost linear. Therefore, we plan to perform tests using new Pentium IV processors with hyper threading technology in order to see if our thread implementation benefits from hyper threading technology that divides one physical processor into two logical processors. Another idea to be implemented in the future is to connect the quadprocessor PC into the same local area network with the rest of the PCs and to modify the decompression algorithm into using the distributed environment.

\section{Acknowledgments}

The authors would like to thank the East Finland Graduate School in Computer Science (ECSE), the Lauri and Lahja Hotinen fund and the Jenny and Antti Wihuri foundation for their financial support.

\section{References}

1. Martin, G., Range Encoding: an Algorithm for Removing Redundancy from a Digitized Message. Video and Data Recording Conference. (1979).

2. Montgomery, C., Runger, G., Applied Statistics and Probability for Engineers. (1994).

3. Mielikäinen, J., Kaarna, A., Toivanen, P. Lossless Hyperspectral Image Compression via Linear Prediction. SPIE's 16th Annual International Symposium on Aerospace/Defense Sensing, Simulation, and Controls. (2002) 600-608. 
4. Airborne visible/infrared imaging spectrometer (AVIRIS) 1997 data. [Online]. Available: http://popo.jpl.nasa.gov/html/aviris.freedata.html.

5. Baizert, P., Pickering, M.R., Ryan, M.J., Compression of hyperspectral data by spatial/spectral discrete cosine transform. Geoscience and Remote Sensing Symposium. 4 (2001) 1859-1861.

6. Mielikäinen, J., Toivanen, P. Improved Vector Quantization for Lossless Compression of AVIRIS Images. European Signal Processing Conference (EUSIPCO). (2002) 495-497.

7. Porter, W., Enmark, H., A system overview of the airborne visible/infrared imaging spectrometer (AVIRIS), Proceedings of SPIE. 834 (1987) 22-31.

8. Vane, G., Green, R., Chrien, T., Enmark, H., Hansen, E., Porter, W., The Airborne Visible/Infrared Imaging Spectrometer (AVIRIS). Remote Sensing Environment. 44 (1993) 127-143. 